\title{
Giant Borderline Phyllodes Tumor With Malignant Presentation: A Case Report
}

\author{
Vidisha Desai $^{\mathrm{a}, \mathrm{c}}$, Vincent Marcucci ${ }^{\mathrm{a}}$, b, Stephanie Steiger ${ }^{\mathrm{a}}$, Denise Johnson-Miller ${ }^{\mathrm{b}}$
}

\begin{abstract}
Phyllodes tumor (PT) is a rare benign tumor with malignant potential. There have been few case reports that have described this condition. We report the case of a 61-year-old woman with a "giant" borderline PT who underwent a modified radical left breast mastectomy with left axillary lymph node dissection, followed by chest wall reconstruction using serratus anterior and pectoralis major muscle flap coverage. A breast tissue measuring $22.5 \times 18.5 \times 12.5 \mathrm{~cm}$ and weighing a total of $5.2 \mathrm{lbs}$ was resected. A case report like this can provide further information on surgical management of the tumor and the importance of an early diagnosis. In addition, PTs are rare to find in patients and this case will further contribute to the literature regarding $\mathrm{PT}$.
\end{abstract}

Keywords: Phyllodes tumor; Breast; Mastectomy; Oncology; Giant

\section{Introduction}

Phyllodes tumors (PTs) of the breast, also referred to as cystosarcoma phyllodes, adenomatous myxoma, and pseudosarcoma adenoma [1], are rare fibroepithelial tumors composed of epithelial and cellular stromal components $[1,2]$. PT accounts for less than $1 \%$ of all diagnosed breast neoplasms in the world [2]. The name "phyllodes" is derived from Greek origin meaning leaflike, as a result of the histological pattern in which they grow. Classically, PT occurs in females during their fourth and fifth decades of life [3]. These tumors have the potential to grow at a significant rate, and may mimic symptoms of other breast neoplasms, particularly if skin ulceration and bleeding occur $[2,3]$. Metastasis to the axillary lymph nodes is uncommon; nevertheless, treatment remains surgical resection [1-3].

Manuscript submitted July 27, 2021, accepted September 1, 2021

Published online September 20, 2021

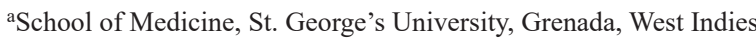
bDepartment of Surgery, Jersey Shore University Medical Center, Neptune, NJ, USA

${ }^{\mathrm{c} C o r r e s p o n d i n g ~ A u t h o r: ~ V i d i s h a ~ D e s a i, ~ D e p a r t m e n t ~ o f ~ S u r g e r y, ~ D i v i s i o n ~ o f ~}$ Breast Surgery, Jersey Shore University Medical Center, 1945 NJ Route 33, Neptune, NJ 07753, USA. Email: Vdesai1@sgu.edu

doi: https://doi.org/10.14740/jcs445
PT can perplex clinicians when determining the appropriate diagnostic and treatment algorithm. The World Health Organization (WHO) currently classifies PT into one of three categories: benign, borderline, or malignant strictly based on mitotic activity in a minimum of 10 high-power field (HPF), the level of cellular stromal atypia and overgrowth, as well as the degree of necrosis and margin appearance [4]. It is important to note that all forms of PT have malignant potential with the possibility of hematogenous spread [3]. PT has a relatively high tendency for local recurrence with an overall survival rate of $>85 \%$ [3]. Herein, we report an interesting case of a rapidly growing, giant borderline phyllodes variant presenting with malignant characteristics.

\section{Case Report}

\section{Investigations}

A 61-year-old female patient with no significant past medical history presented to the emergency department (ED) complaining of bleeding from the skin of the left breast that had been increasing in size for 6 months. Prior to seeking medical advice, she endorsed spontaneous non-bloody discharge, bouts of pain, and ulceration of the skin to the left breast. On presentation, the patient was alert with no complaints of pain and normal vital signs. There was no family history of similar illness reported. On breast examination, the left breast demonstrated hypervascularity, fluctuance, and areas of hyperpigmentation, and was noted to be more than twice the size of the right breast. Routine laboratory workups, including hemoglobin (Hgb) and white blood cell (WBC) count, were within normal limits.

\section{Diagnosis}

Ultrasonography was performed showing a combination of solid and cystic counts with the presence of hypervascularity. However, a neoplastic process could not be ruled out. The patient was discharged and advised to follow-up with a breast surgeon as an outpatient (OP).

At her OP follow-up visit, an incisional biopsy done in the office was inclusive for a diagnostic pathology, prompting an excisional biopsy to be performed. The pathology report of the second biopsy exhibited a well-defined border with a mitotic 


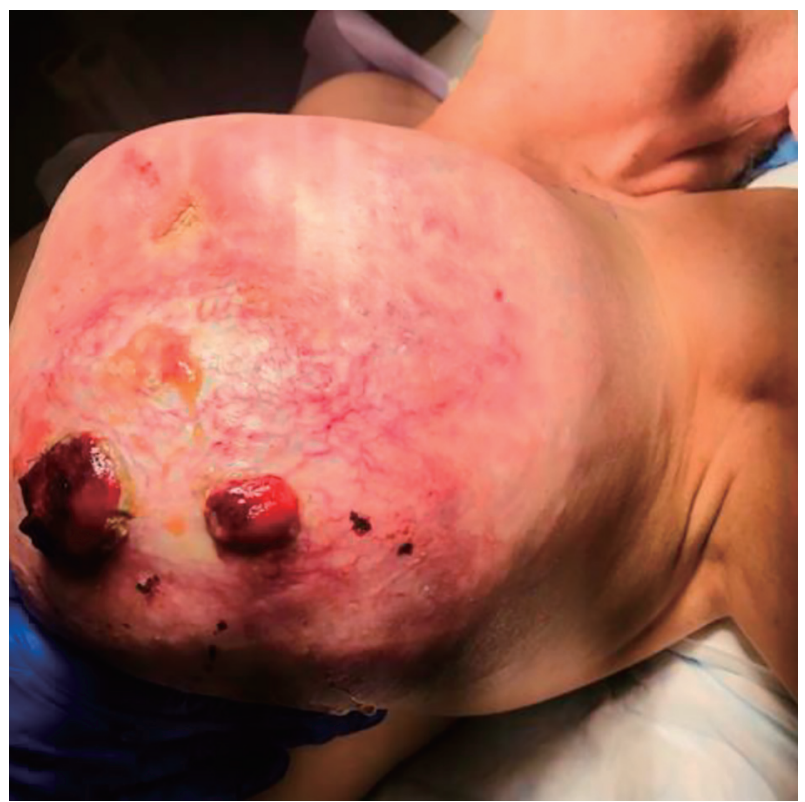

Figure 1. Pre-operative phyllodes tumor of the left breast.

count as high as 5/10 HPF, moderate stromal cellularity, mild cellular atypia, and the absence of necrosis. Histological staining results for calponin, E-cadherin and $\mathrm{Ki}-67 \mathrm{~A}$ were negative. A definitive diagnosis of borderline PT was established. The patient was advised on the treatment options and a conclusion was made with the patient and her family to undergo surgical resection of the breast tumor. A positron emission tomography (PET) scan was done prior to surgery to assist in staging of her disease process. The PET scan did not show evidence of metastatic disease.

\section{Treatment}

The patient underwent an uncomplicated modified radical left breast mastectomy with left axillary lymph node dissection, followed by chest wall reconstruction using serratus anterior and pectoralis major muscle flap coverage. In the operating room (OR), the left breast had necrotic, hemorrhagic tissue present from her previous biopsies with serosanguinous discharge and hypervascularity to the anterior surface (Fig. 1). A breast tissue measuring $22.5 \times 18.5 \times 12.5 \mathrm{~cm}$ and weighing a total of $5.2 \mathrm{lbs}$ was resected (Fig. 2).

\section{Follow-up and outcomes}

Her postoperative course was uneventful and she was discharged home on postoperative day 2 with a Jackson-Pratt (JP) drain in the left hemithorax to be removed at her first OP visit. The patient also had a follow-up appointment with an oncologist at 5 weeks postoperatively. At that time, the patient was recovering well with no new complaints. Observation, surveillance, and clinical evaluation were determined to be the best plan of care for her.

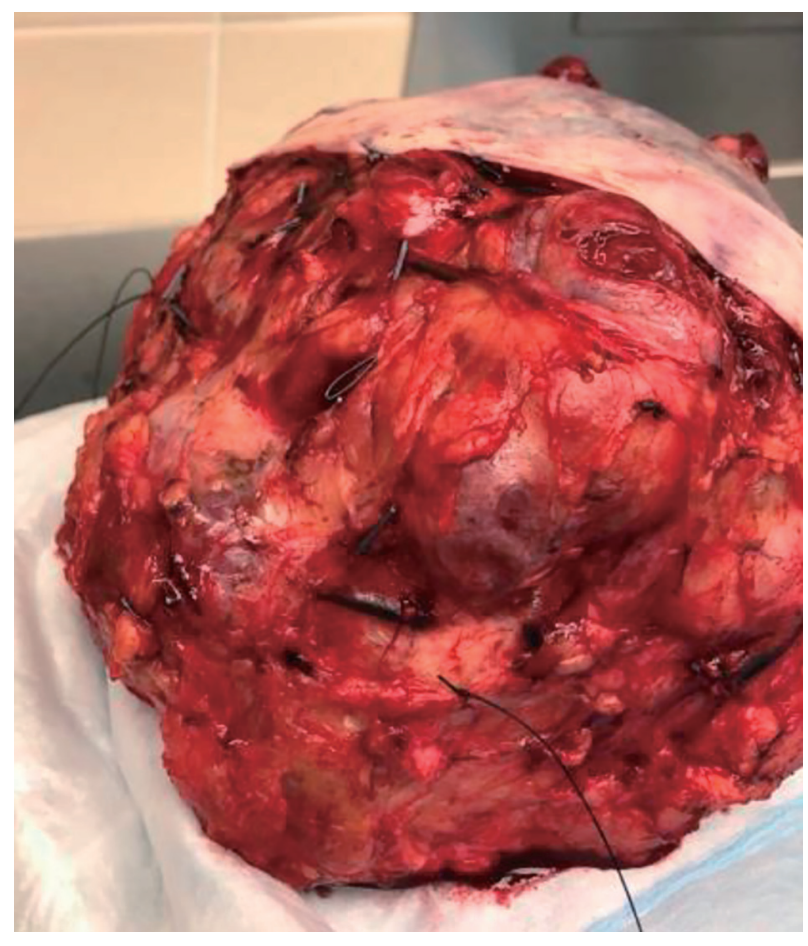

Figure 2. Post-operative gross image of a phyllodes tumor.

\section{Discussion}

The incidence of PT is commonly a result of an incidental finding during a routine breast examination in middle-aged women that can easily be confused with fibroadenomatous growths. While PT is much more common in females, it has been previously reported in male patients in the breast, prostate, urethra, and seminal vesicles [5]. The etiology surrounding PT currently remains unclear with the left breast more frequently affected, similar to our case $[5,6]$. A painless mass with an average size of $4-5 \mathrm{~cm}$ in the breast is the typical presentation for these tumors [6]. However, a PT reaching sizes of more than $20-30 \mathrm{~cm}$ has been documented. Our patient having a tumor size of $22.5 \mathrm{~cm}$ is considered "giant" by clinical standards [7]. The growth of these tumors, regardless of size, is usually not in a vertical growth pattern like many other aggressive, malignant tumors, rather, in a horizontal-radial pattern expressing a mass-effect on the surrounding breast tissue $[3,7]$. The degree of growth with PT can cause thinning of the skin resulting in ulceration, hemorrhagic discharge, and an increased risk of infection.

The majority of PTs are diagnosed as benign (60-70\%) [8]. Borderline phyllodes are diagnosed $<25 \%$ of the time when a benign or malignant neoplasm cannot be distinguished, and malignant tumors are found in approximately $10-20 \%$ of cases [8]. While malignant PTs are rare, there is risk of metastasis, particularly to the lungs and bones [3]. The conclusive diagnostic approach for PT is incisional or excisional biopsy, as well as core needle biopsy [3]. Ultrasonography and mammography have been utilized as preliminary non-invasive diagnostic techniques [9]. Though, ra- 
Table 1. An Algorithm for Differentiating Between Benign, Borderline and Malignant Phyllodes Tumors

\begin{tabular}{llll}
\hline Histological features & Benign & Borderline & Malignant \\
\hline Stromal hypercellularity & Minimal & Moderate & Marked \\
Cellular pleomorphism & Little & Moderate & Marked \\
Mitosis & $0-4 / 10 \mathrm{HPF}$ & $5-9 / 10 \mathrm{HPF}$ & $>10 / 10 \mathrm{HPF}$ \\
Margins & Pushing & $\begin{array}{l}\text { Zone of microscopic invasion } \\
\text { around tumor margins }\end{array}$ & Invasive \\
Stromal pattern & & Heterogenous stromal expansion & Marked stromal overgrowth \\
Heterologous stromal differentiation & Rare & Rare & Not uncommon \\
\hline
\end{tabular}

HPF: high-power field.

diographic imaging cannot definitively differentiate amongst the three forms of PT or from other benign breast conditions [9]. Under histological evaluation, borderline PTs show a greater degree of atypia when compared to benign; the mitotic index is $5-9 / 10$ HPF with moderate stromal cellularity, and areas of hemorrhage and necrosis may be identifiable [4, 10]. Borderline phyllodes have demonstrated the highest rate of recurrence after surgical intervention [10]. A diagnostic pathological algorithm for discerning the type of PT can be seen in Table 1.

First-line treatment for patients diagnosed with PT is surgical resection $[1,3,11]$. According to the National Comprehensive Cancer Network (NCCN) guidelines for breast cancer, the management for PTs $>3.0 \mathrm{~cm}$ is with surgical excision with clear margins of at least $1.0 \mathrm{~cm}$ without axillary staging, regardless of the phyllodes sub-category [1]. Previous literature has demonstrated a recurrence rate of PT as high as $40 \%$ when surgical resection is performed with margins under $1.0 \mathrm{~cm}$ [1]. In contrast to resection with tumorfree margins, the recurrence rate is less than $13 \%$ [1]. Recurrence with PT is particularly higher when the tumor size is larger than $10 \mathrm{~cm}[3,11]$. The use of neoadjuvant and adjuvant therapy with chemotherapeutic agents and/or radiation treatment does not have a clear indication for this pathology $[1,9-11]$; the treatment decision with mastectomy alone for our patient was determined as appropriate. Close follow-up appointments with regular breast examinations and imaging tests are suggested for patients after surgery. Our patient had several risk factors for local recurrence, principally the tumor size, borderline subtype, and the significance of histological findings.

Unlike the well-studied genetic BRCA1 and BRCA2 mutations in breast cancer, there is a lack of scientific literature linking genetic variations to the growth of PT [12]. There have been studies associating mutations in PT53 gene to malignant PT $[12,13]$. Our patient was not tested for the PT53 mutation; however, histological staining for calponin, E-cadherin, and $\mathrm{Ki}-67$ was performed to rule out other breast carcinomas, which all returned negative.

A clear and appropriate diagnostic algorithm is necessary for surgical planning and to prevent the risk of recurrence and reoperation. Biopsy remains the gold standard for diagnosis of PT, while the usefulness of fine needle aspiration remains debatable. Surgical intervention with wide margins is the only curative measure for this breast condition and minimizes the risk of recurrence.

\section{Learning points}

The main learning points of this case report stress the unique clinical picture of a benign borderline PT which can present similar to a malignant neoplasm. An appropriate diagnostic workup is necessary for patients with this presentation to rule out life-threatening sequela. Surgical resection remains the first-line treatment for patients diagnosed with PT.

\section{Acknowledgments}

We are honored to be representing the school. Also, a huge thank you to JSUMC and Dr. Johnson Miller, for giving us the opportunity to write up a case report on one of your patients.

\section{Financial Disclosure}

Funding for this manuscript was received from St. George's University School of Medicine.

\section{Conflict of Interest}

None to declare.

\section{Informed Consent}

Written informed consent was obtained from the patient for publication of this case report and accompanying images.

\section{Author Contributions}

VD, VM, and SS wrote the article. VD and DJM participated in the patient's care. DJM supervised the article writing. All authors read and approved the manuscript, and ensure that this is the case. 


\section{Data Availability}

The data supporting the findings of this study are available from the corresponding author upon reasonable request.

\section{References}

1. Abe H, Teramoto A, Takei Y, Tanaka Y, Yoneda G. Malignant phyllodes tumor of the breast with rapid progression: a case report. Surg Case Rep. 2020;6(1):308.

2. Tan BY, Acs G, Apple SK, Badve S, Bleiweiss IJ, Brogi E, Calvo JP, et al. Phyllodes tumours of the breast: a consensus review. Histopathology. 2016;68(1):5-21.

3. Albalawi IA. A huge phyllodes tumor in the breast: a case report. Electron Physician. 2018;10(6):6951-6955.

4. Tan PH, Ellis I, Allison K, Brogi E, Fox SB, Lakhani S, Lazar AJ, et al. The 2019 World Health Organization classification of tumours of the breast. Histopathology. 2020;77(2):181-185.

5. Karihtala P, Rissanen T, Tuominen H. Male malignant phyllodes breast tumor after prophylactic breast radiotherapy and bicalutamide treatment: a case report. Anticancer Res. 2016;36(7):3433-3436.

6. Takenaka M, Toh U, Otsuka H, Takahashi H, Iwakuma N, Nakagawa S, Fujii T, et al. Giant malignant phyllodes tumor: a case report. Kurume Med J. 2011;58(2):67-72.
7. Tan PH, Thike AA, Tan WJ, Thu MM, Busmanis I, Li H, Chay WY, et al. Predicting clinical behaviour of breast phyllodes tumours: a nomogram based on histological criteria and surgical margins. J Clin Pathol. 2012;65(1):6976.

8. Wang Y, Zhang Y, Chen G, Liu F, Liu C, Xu T, Ma Z. Huge borderline phyllodes breast tumor with repeated recurrences and progression toward more malignant phenotype: a case report and literature review. Onco Targets Ther. 2018;11:7787-7793.

9. Kim JG, Kim SY, Jung HY, Lee DY, Lee JE. Extremely rare borderline phyllodes tumor in the male breast: a case report. Clin Imaging. 2015;39(6):1108-1111.

10. Zhou ZR, Wang CC, Sun XJ, Yang ZZ, Chen XX, Shao $\mathrm{ZM}, \mathrm{Yu} \mathrm{XL}$, et al. Prognostic factors in breast phyllodes tumors: a nomogram based on a retrospective cohort study of 404 patients. Cancer Med. 2018;7(4):1030-1042.

11. Belkacemi Y, Bousquet G, Marsiglia H, Ray-Coquard I, Magne N, Malard Y, Lacroix M, et al. Phyllodes tumor of the breast. Int J Radiat Oncol Biol Phys. 2008;70(2):492500 .

12. Wang Y, Zhu J, Gou J, Xiong J, Yang X. Phyllodes tumors of the breast in 2 sisters: Case report and review of literature. Medicine (Baltimore). 2017;96(46):e8552.

13. Garlet BB, Zogbi L, Lima JP, Favalli PPS, Krahe FD. Recurrent borderline phyllodes tumor of the breast submitted to mastectomy and immediate reconstruction: Case report. Int J Surg Case Rep. 2019;60:25-29. 